\title{
Landscape Design Optimization Model and Simulation Analysis based on Target Contribution Rate Algorithm
}

\author{
Chengyong Shi ${ }^{1,2}$, Pei Zhang ${ }^{1}$, Ying Tang ${ }^{1,2}$ \\ ${ }^{1}$ College of Architecture, Xi'an University of Architecture and Technology, 710311, Xi'an, China \\ ${ }^{2}$ College of Landscape Agriculture and Arts, Northwest Agriculture and Forestry University, \\ Xianyang, 712100, China
} Keywords: Ecological planning, Central park landscape,Multi target contribution rate, Linear
weighted function, Correlation

\begin{abstract}
The paper analyzes the correlation of historical cultural city and central parks in detail. Contribution prior model is established according to multi-objective contribution rate mathematical theory knowledge. Linear weighted sum and function of central park landscape ecological planning contribution is found. In the last part of thesis mathematical model in second part has been used. The correlation of Yulin central park historical cultural city and central park landscape ecological planning is analyzed according to actual of Yulin central park street. Mathematical statistics of removal rate of Yulin central park historical cultural city and decrement of tourist number find correlation of historical cultural city and contribution of central park landscape ecological planning.
\end{abstract}

\section{Introduction}

In ancient times, streets meant traffic road. It was also place for leisure. In the era of no horses and chariots, street was space for organization of community life [1,2]. Streets and roads belong to the space of pedestrian. People can play, shopping, chatting, dating and have fun here. They can complete all activities that need for going shopping. In the age of chariots and horses, conflict between people and chariots began to show up. But the contradiction was not obvious. When it enters into automobile age, the nature of the street completely changed $[3,4]$. People have to risk their lives to go out all day long for disorder caused by human and cars. People walk on the street with the aid of traffic safety island, dedicated pedestrian, traffic signs and management system [5]. They have to endure the noise and automobile exhaust pollution which seriously affect people the fun of shopping.

\section{Exploration Mathematical Model of Historical Cultural City in the Central Park Landscape Ecological Planning}

Historical cultural city are generally ancient streets. Street does not have the phenomenon such as heavy traffic and mutual shading and thus becomes a bright scenery line. Contribution of historical cultural city to Central park landscape ecological planning mainly reflects in appeal to tourists, the degree of quiet and peaceful life and the integrity of landscape planning [6,7]. As for mathematical model for contribution of historical cultural city central park landscape ecological planning, TongChouXue theory can be used to do linear processing. The linear correlation coefficient can be represented as:

$$
\begin{array}{ll}
\text { optF } & (X)=\left(f_{1}(X), f_{2}(X), \cdots, f_{p}(X)\right)^{T} \\
\text { s.t. } & g_{i}(X) \geq 0 \\
& h_{j}(X)=0
\end{array}
$$




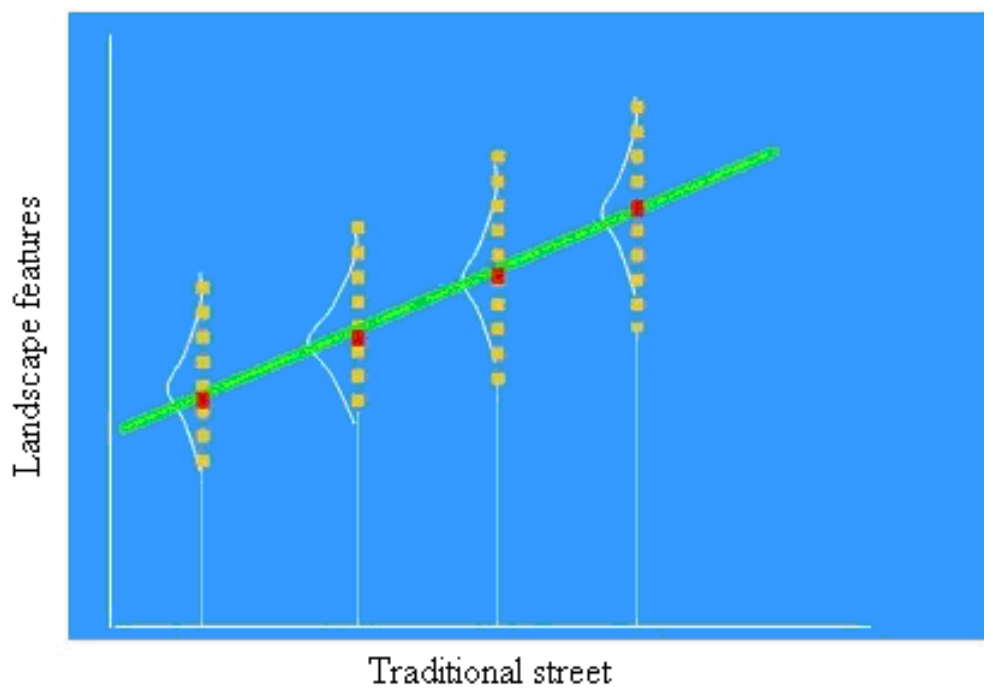

Fig. 1 The historical cultural city and central park landscape ecological planning

Each value of the historical cultural city and distribution of central park landscape ecological planning determine expectation or average, which is called conditional expectation or average value of central park landscape ecological planning [8]. Relevant model shown in Figure 2 can be expressed in mathematical diagram as shown in Figure 2.

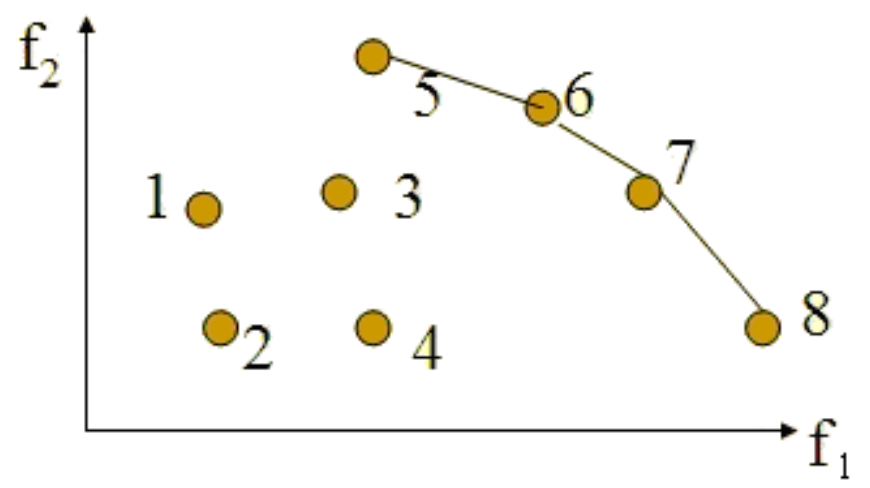

Fig. 2 Mathematical model diagram

As for the solutions to any two schemes, its size of the contribution can be judged as long as to compare their respective target through the mathematical model shown in Figure 3.

The contribution of historical cultural city for the central park landscape ecological plannings can be expressed by utility function [9]. Assumes that target utility function of contribution rate as follows:

$$
U(x)=U\left(f_{1}, f_{2}, \cdots, f_{p}\right) .
$$

Assumes that $a_{i j}=f_{i}\left(x_{j}\right)$, contribution function of $\mathrm{f}$ historical cultural city characteristic is:

$$
U\left(x_{j}\right)=U\left(a_{1 j}, a_{2 j}, \cdots, a_{p j}\right) .
$$

Prior contribution model structure of historical cultural city to central park landscape ecological planning can be expressed as follows:

$$
\begin{array}{ll}
\operatorname{ord} F(X)=\left(f_{1}\left(X_{1}\right), f_{2}\left(X_{2}\right), \cdots, f_{p}\left(X_{p}\right)\right)^{T} \\
\text { s.t. } & g_{i}(X) \geq 0 \\
& h_{j}(X)=0
\end{array}
$$

Assuming that $f_{1}\left(X_{1}\right), f_{2}\left(X_{2}\right), \cdots, f_{p}\left(X_{p}\right)$ has the same dimension, according to certain rules, the same weight $\omega_{i}$ is given to $f_{i}$. Linear weighting and contribution function of historical and cultural city to Central park landscape ecological planning can be expressed as follows: 


$$
U(X)=\sum_{i=1}^{p} \omega_{i} f_{i}(X)
$$

Multi-contribution can be written as function of single contribution as follows:

$$
\begin{aligned}
& \max U(X)=\sum_{i=1}^{p} \omega_{i} f_{i}(X) \\
& \text { s.t. }\left\{\begin{array}{l}
g_{i}(X) \geq 0 \\
h_{j}(X)=0
\end{array}\right.
\end{aligned} \text {. }
$$

As shown in formula (4), through the node verification of digital certificate, any state of data integrity monitoring probability $a_{p_{i} \cdot p_{t}}$ from state $p_{0}$ to state $p_{i}$. Any state of data integrity is the probability $a_{s_{i} \cdot s_{0}}$ from state $s_{i}$ to state $s_{0}$. By controlling the probability of the test data, it ensures the integrity of teachers' confidential information.

\section{Research of the Contribution of Historical Cultural City in the Central Park Landscape Ecological Planning}

There are a lot of historical nd cultural city in Yulin central park area. Street has rich historical and cultural relics, developed foreign trade, and rich folk life [10]. Historical cultural city in Yulin central pPark gave birth to the unique local characteristic culture and contributed to a unique central park landscape ecological plannings.

Main features of historical cultural city contribution rate centers on correlation of historical cultural city and central park landscape ecological planning. The greater contribution rate of historical cultural city for the central park landscape ecological planning, the higher related degree. This paper combines with contribution of historical cultural city to central park landscape ecological planning mathematical model in second part and makes a mathematical statistics analysis of removal rate and the characteristic street since 2008 and the number of tourists in Yulin central park area though survey. It makes curve drawing for final statistical results. Finally the related curves are obtained as shown Figure 3.

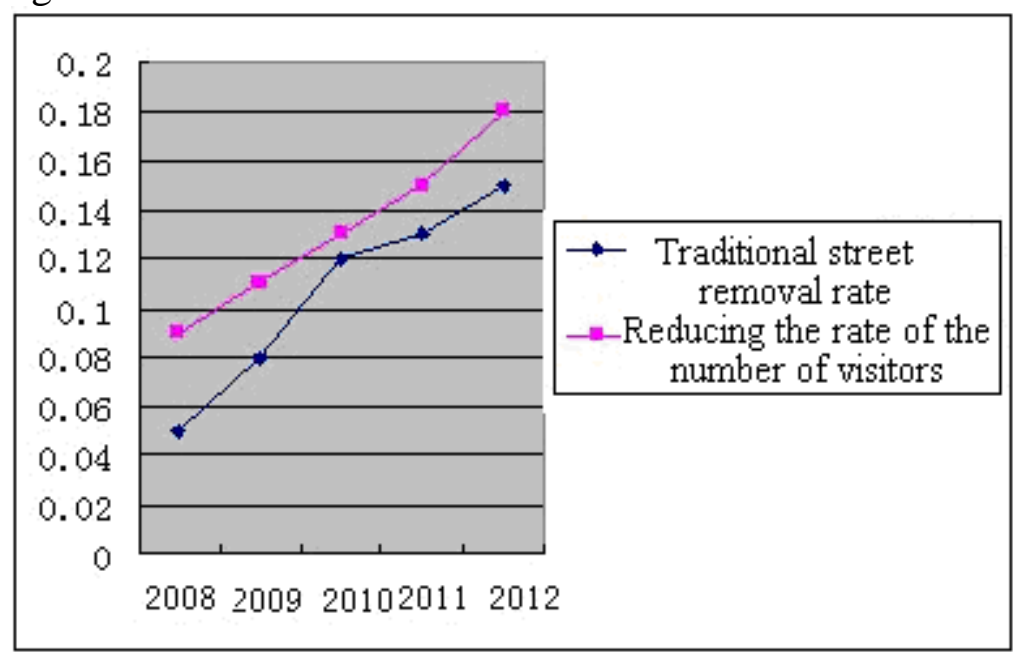

Fig. 3 historical cultural city's contribution to the central park landscape ecological plannings

Contribution of historical cultural city to Central park landscape ecological planning is very high according to the Figure 6 . This mainly reflects in the number of visitors. As the historical cultural city removal rate increasing in Figure 6, the number of visitors shows downward trend year by year. In 2008, the removal rate of historical cultural city is only 5\%. In 2012, removal rate is $15 \%$, which is increased by three times. Tourists gradually reduce as the historical cultural city removal rate increased year by year in term of the number of tourists. In 2008, the number of visitors was reduced by $9 \%$. In 2012, the number of visitors was reduced by $18 \%$. Analysis in this paper fully validates 
contributions that historical cultural city make to central park landscape ecological planning, which provides central park landscape ecological planning for scenic area landscape design.

\section{Summary}

Contribution prior model and linear weighted sum and function of contribution Central park landscape ecological planning have been established based on multi-objective mathematical theory knowledge. A detailed research was made in contribution of historical cultural city to central park landscape ecological planning. This paper takes historical cultural city in Yulin central park area as an example and makes an analysis of humanities and regional history central park landscape ecological planning in Yulin central park, finding that the extent of the destruction of historical cultural city is larger. Guangfu road was widened $21 \mathrm{~m}$ which caused great damage to original traditional central park landscape ecological planning. At the end of the paper, it uses method of investigation statistics combining with mathematical statistics analysis tools to analyze the relationship between historical cultural city and central park landscape ecological planning, finally finding that the number of landscape tourist reduced gradually with removal rate of historical cultural city increasing, in which it was decreased by $18 \%$ in 2012, it fully illustrates the contribution of historical cultural city to central park landscape ecological planning and provides a theoretical reference for landscape street design.

\section{References}

[1] M.H. Zhou, X.Z. Meng and J. Xu. Analysis and Countermeasures of Villages Landscape Problem in Gaomi Shandong Province. Heilongjiang Agricultural Sciences, 2012(4): 98-102.

[2]H. Wang, X.L. Tang, and X.W. Zhang. Village central park landscape ecological plannings and Integration. BeiJing: China Forestry Publish, 2012: 312-320.

[3]X. H. Chen, X.L. Zhang. Research Overview of Foreign Rural Community Change. LiuAn: WanXi College Journal. Research Overview of Foreign Rural Community Change. AnHui: WanXi College Journal Editorial Office, 2013: 10-12.

[4](America)Esther, Charles worth. The Edge of the City: a Case Study of Contemporary Urbanization. H.S. Xia, Q. Liu, Translate. Beijing: China Machine Press, 2013: 112-123.

[5](America)Oliver, Durham. The Horizon of the City. M.S. Ye, X.H. Ni, Translate. Beijing: China Building Industry Press, 2012: 78-89.

[6]W. Chen. New Rural Landscape: Rural Landscape Planning Theory and Method. Beijing: China Electric Power Press, 2012: 172-180.

[7]X.Q. Zhou. The west rural landscape research progress. Zhengzhou: Areal Research and Development, 2012(3):85-86.

[8](America) Esther., Chad's right. The Edge of the City: a Case Study of Contemporary Urbanization. H.S. Xia, Q. Liu, Translate. Beijing: China Machine Press, 2012(1): 310-320.

[9](Japan)Ashihara Yoshinobu. Aesthetics of the Street. P.T. Yin, Translate. Beijing: China Building Industry Press, 2012: 45-50.

[10](America)Alan b, jacobs. The Great Street. Y.J. Wang, Q.Y. Jin, Translate. Beijing: China Building Industry Press, 2013: 630-638. 